\title{
Coping through coaching: Co-active life coaching as a method for stress and anxiety management
}

\author{
Rebecca Fried
}

University of Western Ontario

\section{Stress, Anxiety, and Family Physicians}

Family physicians (FPs) are often the first point of contact for health-related issues. With respect to mental illness, which is inclusive of stress and anxiety, FPs often serve as a conduit and refer patients to other specialists. Approximately $20 \%$ of Canadians are impacted by mental illness in their lifetime. ${ }^{1}$ Thus, it is vital for FPs to be familiar with a variety of management options. In 2011, 23.6\% of Canadians aged 15 and older reported feeling most of their days were extremely or quite a bit stressful. ${ }^{2}$ Anxiety affects $5 \%$ of the population, causing mild to severe impairment in daily life. ${ }^{1}$ Furthermore, fewer than $33 \%$ of individuals receive sufficient help. ${ }^{3}$ These statistics are clear indicators that stress and anxiety (hereafter referred to as stress/anxiety) are a concern. As a result, an approach that could help FPs support patients is Motivational Interviewing via Co-Active Life Coaching (MI-via-CALC).

\section{MI-via-CALC: Defined and Described}

MI-via-CALC provides a platform to manage stress/anxiety on an individual basis. MI-via-CALC is an approach in which the coach and client operate as a team, in service of the client identifying goals and actions toward making behaviour changes. ${ }^{4,5}$ Stress/anxiety is the result of how an individual reacts to stressors; and research suggests that individuals suffering from stress/anxiety are often ambivalent toward change and feel uncomfortable in changing familiar behaviours (i.e. habitual stress responses). ${ }^{6}$ The components of MI-via-CALC help clients to feel empowered and capable of making behaviour changes. A cornerstone of MI-viaCALC is to hold the client naturally creative, resourceful, and whole, as opposed to being incapable of change. ${ }^{4,5}$ A positive attribute of the model is that it encompasses a client's whole life, appreciating that one aspect of life may impact others. ${ }^{4,5}$ For instance, an upsetting argument may also impact the individual's exercise choices, which in turn can further impact his/her stress experience. MIvia-CALC focuses on 'where is the person now, and where does he/she want to be.' It does not explore the past to understand a client's current situation. ${ }^{4,5} \mathrm{MI}$-via-CALC is often done over-the-phone, at a mutually convenient time for the coach and client. This is an important feature of MI-via-CALC, as individuals who perceived their mental health needs were unmet reported the reason was due to personal circumstances, such as being too busy. ${ }^{7}$ Other positive elements are balance and perspectives. ${ }^{4,5}$ Balance is a coaching tool that emphasizes that the client always has a choice in how he/she acts. ${ }^{4,5}$ Perspectives refer to looking at a situation from multiple viewpoints. ${ }^{4,5}$ FPs should consider this technique for stress/anxiety, as it permits treatment to be tailored to an individual's needs.

\section{Research Findings}

There is limited research supporting MI-via-CALC for stress/ anxiety. However, findings from other studies suggest this approach is a viable option for stress/anxiety. MIvia-CALC interventions for smoking cessation and obesity have demonstrated promising results with participants able to reduce smoking rates and waist circumference, respectively. ${ }^{8,9}$ Participants from both studies, albeit rather different, noted through interviews that their levels of stress had been reduced due to the coaching. ${ }^{8,9}$ Quantitatively, this reduction in stress levels was consistent with increases in the participants' levels of self-efficacy and self-esteem. ${ }^{8,9}$ Currently, research is being conducted observing the impact of MI-via-CALC on university students suffering from stress. Studies are also being conducted in the field of neuroscience to comprehend coaching's effect on the brain, which is crucial to understanding how coaching affects mental health. ${ }^{10}$ Research to date has provided an evidence base supporting MI-via-CALC as a management option that FPs can recommend to their patients. 


\section{The Other Side: Limitations}

There are several limitations surrounding MI-via-CALC. Hiring a coach may be expensive with sessions costing hundreds, and sometimes thousands, of dollars. Coaching is not a regulated profession and fees are not consistent. Coaches may charge what they desire, potentially causing financial strain for those seeking a coach. MI-via-CALC is a unique approach, and coaches must be rigorously trained and certified with CALC skills. There are many coaches who claim to provide health and wellness coaching similar to MIvia-CALC, but lack sufficient training. Due to limited research, coaching may not be suitable for more severe forms of mental illness. Thus, FPs need to ensure that patients are referred to highly trained coaches, and coaches that are able to work out a financial arrangement with the client.

\section{Conclusion}

MI-via-CALC is a promising intervention for stress/anxiety management. This approach contains many components intended to address patients on an individual basis; this is crucial, considering the individualistic nature of stress/ anxiety. Based on previous and emerging research, there is evidence to support MI-via-CALC as a worthy method of addressing stress/ anxiety. Thus, FPs should suggest MI-viaCALC as a management option for patients.

\section{References}

1. Canadian Mental Health Association. Fast facts about mental illness [Internet]. Canada: Canadian Mental Health Association; 2014 [updated 2014; cited 2014 Mar 13]. Available from: http://www.cmha.ca/mental_ health/what-is-mental-illness/\#.Ut8FIHnOCXO

2. Statistics Canada. Perceived life stress [Internet]. Canada: Government of Canada; 2011 [updated 2013 Dec 11; cited 2014 Jan 13]. Available from: http://www.statcan.gc.ca/pub/82-625-x/2012001/article/11666-eng.htm

3. Sunderland A, Findlay LC. Perceived need for mental health care in Canada: Results from the 2012 Canadian Community Health Survey - Mental Health. Health Reports. 2013;24(9):3-9.

4. Kimsey-House H, Kimsey-House K, Sandahl P, Whitworth, L. Co-active coaching: Changing business, transforming lives. Third edition. Boston: Nicholas Brealey Publishing; 2011.

5. Irwin, JD, Morrow, D. Health promotion theory in practice: An analysis of co-active coaching. International Journal of Evidence Based Coaching and Mentoring. 2005;3(1):29-38.

6. Westra HA. Motivational interviewing in the treatment of anxiety. New York: The Guilford Press; 2012.

7. Statistics Canada. Canadian community health survey: Mental health, 2012 [Internet]. Canada: Government of Canada; 2013 [updated 2013 Sep 18; cited 2014 Jan 13]. Available from: http://www.statcan.gc.ca/dailyquotidien/130918/dq130918a-eng.htm

8. Mantler T, Irwin JD, Morrow D. Assessing motivational interviewing through co-active life coaching tools as a smoking cessation intervention: A demonstration study. International Journal of Evidence Based Coaching and Mentoring. 2010;8(2):49-63

9. Newnham-Kanas C, Irwin JD, Morrow D. Co-Active life coaching as a treatment for adults with obesity. International Journal of Evidence Based Coaching and Mentoring. 2008;6(2):1-12.

10. Rock D, Page L. Coaching with the brain in mind. Hoboken: John Wiley \& Sons, Inc; 2009.

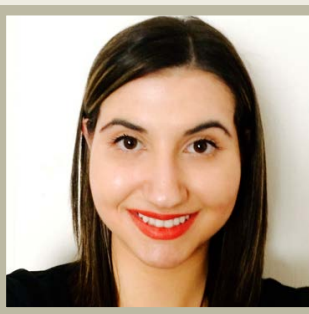

\section{Rebecca Fried}

Rebecca Fried is a second year MSc student at the University of Western Ontario in Health and Rehabilitation Sciences, Health Promotion. Rebecca's research focuses on the impact of Motivational Interviewing and Co-Active Life Coaching (or MI-via-CALC) on university students suffering from stress and anxiety. In September 2014, Rebecca will be continuing her studies at Western in the PhD program. 